\title{
IMPACT OF THE COVID-19 PANDEMIC ON RADIOGRAPHY \& RADIOTHERAPY PRACTICE IN MALAYSIA
}

\author{
MOHD IZUAN IBRAHIM ${ }^{1}$, RAHMAHWATY AKIB ${ }^{2}$ and MOHD FARIHAN JAFFAR ${ }^{3}$ \\ ${ }^{1}$ Diagnostic Imaging \& Radiotherapy Program, Centre for Diagnostics, \\ Therapeutics \& Investigative (CODTIS), Faculty of Health Sciences, \\ Universiti Kebangsaan Malaysia, 50300 Kuala Lumpur, Malaysia \\ ${ }^{2}$ Radiology Department, Hospital Kuala Lumpur, 50586 Kuala Lumpur, Malaysia \\ ${ }^{3}$ Radiotherapy \& Oncology Department, National Cancer Institute, \\ 62250 Wilayah Persekutuan Putrajaya, Malaysia \\ *E-mail: izuan@ukm.edu.my
}

Accepted 23 September 2021, Published online 30 November 2021

\begin{abstract}
This study aimed to evaluate the COVID-19 pandemic impact on radiography and radiotherapy practice in Malaysia. An online cross-sectional survey was conducted from July to October 2020 among radiographers. The questionnaire was adapted from the previous study. A total of 214 (female, $n=148$ and male, $n=66$ ) responses were received comprising $79.9 \%$ diagnostic and $20.1 \%$ therapeutic radiographers across Malaysia. The data were analyzed using IBM SPSS Version 25.0. The results show that $71 \%$ agree that the radiographers are part of the major frontline and $50.9 \%$ agree diagnostic radiographer's workload increases. Besides, $77.1 \%$ and $71.9 \%$ of them are very aware of the COVID-19 virus transmission and infection control principle, respectively. Furthermore, 59.8\% stated that adequate personal protective equipment (PPE) was provided by the department while $57 \%$ receive adequate training in handling patients during the pandemic. There were $66.5 \%$ and $28.6 \%$ of diagnostic radiographers and therapeutics radiographers who receive specific training in facing COVID-19 patients. During the pandemic, only $29 \%$ feel frequently stressed about work and $62.1 \%$ sometimes. The significant association $\chi^{2}(p=0.0002)$ exists between radiographers and the perception of stress. Most of the radiographers rate their level of stress between 5 to 8 on a scale of 1 (no stress) to 10 (highly stress). In conclusion, increasing workload during the pandemic increase the level of stress among radiographers. However, adequate PPE, a good work rotation schedule, and training will help them facing the pandemic.
\end{abstract}

Key words: COVID-19, radiography, radiotherapy, stress, workload

\section{INTRODUCTION}

COVID-19 is an ongoing pandemic affecting worldwide. The disease was first reported in China and caused severe respiratory symptoms such as fever, cough, and breathing difficulty. A more serious respiratory illness that might involve was lung failure and pneumonia. This outbreak of the respiratory disease was caused by the novel coronavirus disease (COVID-19) according to a study conducted by Zhu et al. (2019). The number of cases has soared rapidly ever since the outbreak in China. As of December 2020, the disease has affected more than 6.2 million individuals and resulted in more than 1.5 million deaths worldwide. Moreover, the data from

* To whom correspondence should be addressed. the Ministry of Health Malaysia recorded 75306 total cases with 388 deaths.

COVID-19 has triggered enormous human casualties and economic loss posing a global threat. Healthcare workers such as doctors, nurses, and radiographers who are in close contact with a patient are at a high chance of exposure to the virus. The most common psychological reactions to the COVID19 pandemic are fear and anxiety-related symptoms such as emotional stress (Wong \& Sam, 2011). The psychological effect of the COVID-19 pandemic is not only due to the disease itself but also due to the changing of daily activities to adapt to the new norm and the social isolation caused by the restricted movement control order (Wong \& Sam, 2010). For healthcare workers like radiographers, the pandemic will expose them to the stress condition for the long- 
term, which could impact their health (Dewey et al., 2020) and lead to burnout due to increased workload, role conflicts, and reduced job resources (Shoji et al., 2015).

The consequences that COVID-19 has had on the radiography and radiotherapy practice remains to be seen, as hospitals strive to figure out the new normal as nonessential imaging and radiotherapy procedures were postponed. However, imaging procedures especially Computed Tomography (CT) and chest radiography play a crucial role in the preliminary diagnosis of COVID-19 (Kooraki et al., 2020). The utilization of imaging modalities will remain important to the early diagnosis of COVID19 (Zervides et al., 2020). Thus, radiographers are professionals who are expected to be in the front line of a clinical setting to perform imaging procedures.

From the previous pattern of a patient infected by the COVID-19, the cases were still increasing and the future prediction of the number of patients is still unclear. This situation might give some impact not only on the doctors and nurses but also other healthcare workers such as diagnostic and therapeutic radiographers. Although there are many studies on the impact of COVID-19 on doctors and nurses, there is not any study conducted to evaluate the impact of COVID-19 on both diagnostic and therapeutic radiographers.

Thus, this preliminary survey aims to assess radiographers' opinions on the impact the COVID19 pandemic had on their practice. Furthermore, the radiographer's knowledge of COVID-19 transmission, infection control, and availability of personal protective equipment (PPE) and profile of COVID-19 related stress was also studied.

\section{MATERIALS AND METHODS}

\section{Study respondents}

A preliminary cross-sectional study was conducted for a period of four months, which was from July to October 2020. Convenience sampling was used because of its convenience inaccessibility and proximity. The respondents consisted of diagnostic radiographers and therapeutics radiographers. The sample size was estimated by using Raosoft, Inc. sample size calculator. The calculator used a marginal error of $5 \%$, the confidence level of $95 \%$, an estimated Malaysian radiographer's population size of 3000 , response distribution of $50 \%$ and the estimated sample size was 341. Meanwhile, all the respondents were asked for consent before the implementation of the study. Nuclear medicine radiographers and trainee radiographers were excluded from this survey.

\section{Questionnaire}

The questionnaire was adapted from the current study by Akudjedu et al. (2020) with the study titled impact of the COVID-19 pandemic on radiography practice: findings from a UK radiography workforce survey. The primary construct of this questionnaire was 1 . Socio-demographics 2 . The impact of the pandemic at the workplace 3 . Infection prevention and control and 4. COVID-19 related stress. The survey was distributed online using Google forms in the English language. The readability indices demonstrate an easily understood questionnaire (Flesch Kincaid Grade Level $=7$ and Coleman Liau Index $=12.3$ ). A pilot study was performed by distributing this questionnaire to 15 radiographers. This questionnaire was found to be valid and reliable with Cronbach's alpha of 0.925 , indicating a high internal consistency.

\section{Statistical analysis}

All statistical analyses were carried out by using IBM SPSS Version 25.0. The descriptive statistic in terms of frequency and percentage were performed for all the questions. The Chi-Square test was applied to test the relationship between categorical variables. Statistical significance was set at a $p$-value of less than 0.05 .

\section{RESULTS AND DISCUSSION}

\section{Demographic data}

This four-month survey has collected 214 valid responses (148 female \& 66 male) comprising 79.9\% diagnostic and $20.1 \%$ therapeutic radiographers across Malaysia. $62.8 \%$ of respondent's data has been collected from the estimated sample size $(214 / 341)$. Most of the respondents were aged between $18-29$ years old $(50.0 \%)$ and $30-39$ years old (43.9\%). In terms of the workplace setting, $71.9 \%$ of them were from government hospitals, $14.0 \%$ from private hospitals, $9.4 \%$ from the clinic, and $4.7 \%$ from university-based hospitals. The respondents work as a radiographer in Kuala Lumpur (39.3\%), Selangor $(16.8 \%)$, Perak (15\%), Penang (6.6\%), Putrajaya (6.5\%), Johor (4.7\%), Sabah (3.7\%), Kelantan (2.3\%), Sarawak (1.4\%), Kedah (0.9\%) and each $0.5 \%$ from Pahang, Melaka and Terengganu. Table 1 summarises the socio-demographic background of the respondents.

\section{The perspective of radiographers on the impact of COVID-19 on practice}

A higher proportion of respondents (124/214; $57.4 \%)$ strongly agree and $(29 / 214 ; 13.6 \%)$ admit that radiographers are a part of the main frontline 
Table 1. Socio-demographic characteristics of the respondents $(n=214)$

\begin{tabular}{lcc}
\hline Variables & $\begin{array}{c}\text { Frequency } \\
(\mathrm{n})\end{array}$ & $\begin{array}{c}\text { Percentage } \\
(\%)\end{array}$ \\
\hline Gender & & \\
Male & 66 & 30.8 \\
Female & 148 & 69.2 \\
& & \\
Radiographer & & \\
Diagnostic & 171 & 79.9 \\
Therapeutic & 43 & 20.1 \\
& & \\
Age range & & \\
18-29 & 107 & 50.0 \\
$30-39$ & 94 & 43.9 \\
$40-49$ & 10 & 4.7 \\
$50-59$ & 1 & 0.5 \\
$>60$ & 2 & 0.9 \\
& & \\
Workplace setting & 154 & 71.9 \\
Government hospital & 10 & 4.7 \\
$\quad$ University hospital & 30 & 14.0 \\
Private hospital & 20 & 9.4 \\
Clinic & & \\
\hline
\end{tabular}

healthcare management team in response to COVID19. Besides, 12/214 (5.6\%) disagree, 37/214 (17.1\%) strongly disagree and the rest $12 / 214$ respondents feel neutral. The front-liners or health care workers comprise medical staff such as doctors, nurses and their assistants, radiographers, paramedics, ambulance staff, hospitals porters, physicians, medical officers, graduated medical officers, medical experts, pathologists, police, and others (Tan, 2020). The frontline healthcare considers the higher risk to get infected when dealing with COVID-19 patients. Compared with the public, frontline healthcare workers were at increased risk for reporting a positive COVID-19 test (Nguyen et al., 2020).

The workload of 109/214 (50.9\%) respondents was reported as increasing during the COVID-19 outbreak, whereas irregular pattern workload was $62 / 214(29.0 \%)$, no change in workload was $26 / 214(12.1 \%)$, and only $17 / 214(7.9 \%)$ reported as decreasing workload. A chi-square test of independence was carried out to determine the relationship between diagnostic radiographers and therapeutic radiographers with the impact of the workload. The relationship between these variables was significant, $\chi^{2}(1, \mathrm{~N}=214)=157.8, p=0.001$. The increasing and irregular workload mostly involved the diagnostic radiographers with $98.2 \%$ and $74.2 \%$ respectively. The workload among radiographers might be due to the standard operating procedure to deal either with COVID-19 patients or suspected COVID-19 patients. Healthcare workers who encountered COVID-19 patients, were subjected to more workload compared to those who had no contact with COVID-19 patients at the workplace (Shoja et al., 2020). Moreover, a study from Zervides et al. (2020) reveals an increase in the working hour and the average examination time in the radiology department. Figure 1 demonstrates the impact on workload among diagnostic and therapeutic radiographers.

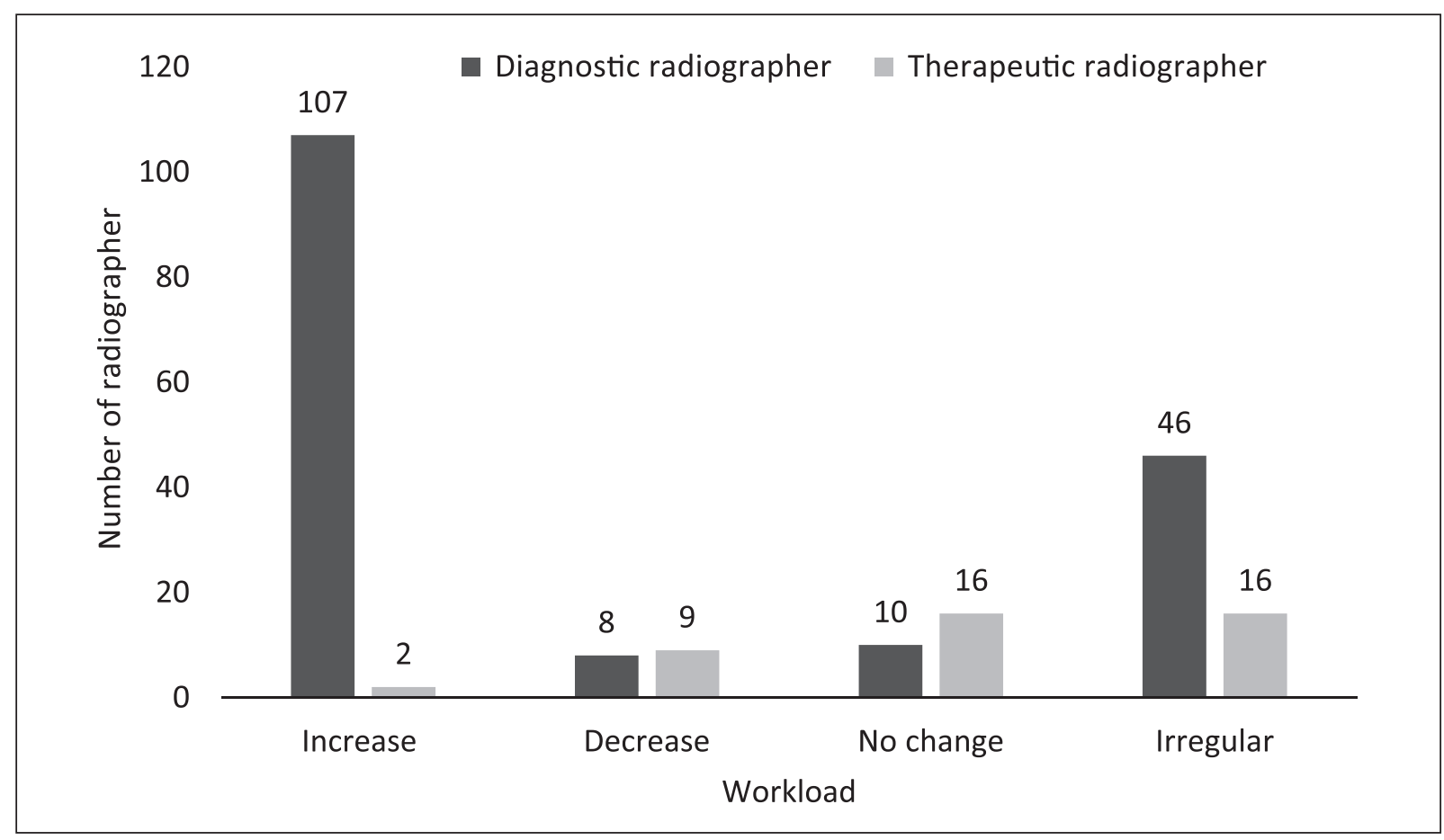

Fig. 1. Impact on workload among diagnostic and therapeutic radiographers during the COVID-19 pandemic. The relationship between radiographers and the workload was significant, $\chi^{2}(1, \mathrm{~N}=214)=157.8, p=0.001$. 
A total of $43.9 \%$ of radiographers had to use other imaging modalities apart from the ones used for their daily work after the COVID-19 outbreak, whereas $56.1 \%$ used their routine imaging modalities. There is an increase in chest radiography and computed tomography scanning leading to the change of the routine working timetable among radiographers (Akudjedu et al., 2020).

Radiographers understanding of COVID-19 infection and availability of personal protective equipment (PPE)

Most radiographers admit that they have a great understanding of how the COVID-19 virus is transmitted, with $52.8 \%$ strongly agree, $23.6 \%$ agree, $2.8 \%$ neutral, $6 \%$ disagree and $14.8 \%$ strongly disagree. Furthermore, 39.4\% (strongly agree) and $31.9 \%$ (agree) respondents believed that their understanding of infection prevention as a radiographer is sufficient to deal with the COVID-19. Only $6.5 \%$ (neutral), $8.3 \%$ (disagree) and $13.9 \%$ (strongly disagree) were lack of confidence to face the COVID-19 based on their understanding of infection prevention. Malaysians have an adequate level of knowledge on COVID-19 and are generally optimistic in their outlook on overcoming the COVID-19 pandemic (Azlan et al., 2020). Continued professional education is advised among healthcare workers to improve knowledge and understanding of COVID-19 (Olum et al., 2020). Table 2 summarises the radiographer's agreement on the understanding of COVID-19.

More than half of radiographers (128/214) strongly agreed $(35.2 \%)$ and agree $(24.1 \%)$ that their department has provided adequate personal protective equipment (PPE) for work during the COVID-19 outbreak, whereas $14.4 \%$ neutral, $12.5 \%$ disagree and $13.9 \%$ strongly disagree. PPE is very crucial to prevent the infection of the virus to the radiographers. A healthcare professional with a good mask, face shield, and gloves should be able to get most of the protection if the conduct is proper (Das \& Rajalingham, 2020). In terms of specific training provided by the department, $123 / 214(57.5 \%)$ had undergone specific training, whereas the rest $91 / 214(42.5 \%)$ does not have any specific training as preparation handling patients during the COVID19 outbreak. There were $66.5 \%$ of diagnostic radiographers who underwent specific training, while only $28.6 \%$ of therapeutic radiographers have been trained to face the COVID-19 patients.

\section{Profile of COVID-19 related Stress, its impact, and available support systems}

The level of stress which was assessed through the question of either the radiographers feel stressed about their work lately due to the COVID-19 outbreak shows that from 171 diagnostic radiographers, 55 of them admit they are stress, 7 have no stress and 109 sometimes have some stress feeling. Besides that, from 43 therapeutic radiographers, 8,11 , and 24 of them either feeling stress, no stress, or sometimes feeling the stress, respectively. The association exist between radiographers and the perception of stress and it was significant, $\chi^{2}(1, \mathrm{~N}=214)=21.4$, $p=0.000023$. Figure 2 shows the bar chart in the percentage of their perception of stress during the pandemic in the workplace. Moreover, based on Likert-scale from 1 (no stress) to 10 (highly stress) regarding the workforce during the COVID-19 outbreak, 19 radiographers rate the highest level of stress (10), 11 rates as 9, 44 rates as 8,48 rates as 7, 26 rates and 6 and 27 rates as 5 (moderate stress). Only 39 rates the level of stress between 1 to 4 . Figure 3 shows the frequency of radiographers and the stress rating scale from 1 to 10 . The stress might relate to the higher workload during the COVID-19 outbreak. The higher workload was linked to worse mental health and higher burnout (Zhou \& Zhang, 2020). Provision of appropriate PPE and work rotation schedules to enable adequate rest in facing longlasting disasters such as the COVID-19 pandemic

Table 2. Summary of the radiographer's understanding of COVID-19 transmission, infection control, and availability of personal protective equipment (PPE)

\begin{tabular}{|c|c|c|c|c|c|c|}
\hline \multirow{2}{*}{ No. } & \multirow{2}{*}{ Question } & \multicolumn{5}{|c|}{$\mathrm{n}(\%)$} \\
\hline & & SA & A & $N$ & D & SD \\
\hline 1 & $\begin{array}{l}\text { I have a great understanding of the COVID-19 } \\
\text { transmitted. }\end{array}$ & $\begin{array}{c}114 \\
(53.3)\end{array}$ & $\begin{array}{c}51 \\
(23.8)\end{array}$ & $\begin{array}{c}6 \\
(2.8)\end{array}$ & $\begin{array}{c}13 \\
(6.1)\end{array}$ & $\begin{array}{c}30 \\
(14.0)\end{array}$ \\
\hline 2 & $\begin{array}{l}\text { My understanding of the infection prevention as a } \\
\text { radiographer is adequate to deal with COVID-19. }\end{array}$ & $\begin{array}{c}85 \\
(39.7)\end{array}$ & $\begin{array}{c}69 \\
(32.2)\end{array}$ & $\begin{array}{c}14 \\
(6.5)\end{array}$ & $\begin{array}{c}18 \\
(8.4)\end{array}$ & $\begin{array}{c}28 \\
(13.1)\end{array}$ \\
\hline 3 & $\begin{array}{l}\text { My facility has made available adequate personal } \\
\text { protective equipment (PPE) for working during } \\
\text { the COVID-19 pandemic. }\end{array}$ & $\begin{array}{c}76 \\
(35.5)\end{array}$ & $\begin{array}{c}52 \\
(24.3)\end{array}$ & $\begin{array}{c}31 \\
(14.5)\end{array}$ & $\begin{array}{c}27 \\
(12.6)\end{array}$ & $\begin{array}{c}28 \\
(13.1)\end{array}$ \\
\hline
\end{tabular}

$\mathrm{SA}=$ strongly agree, $\mathrm{A}=$ agree, $\mathrm{N}=$ neutral, $\mathrm{D}=$ disagree, $\mathrm{SD}=$ strongly disagree . 


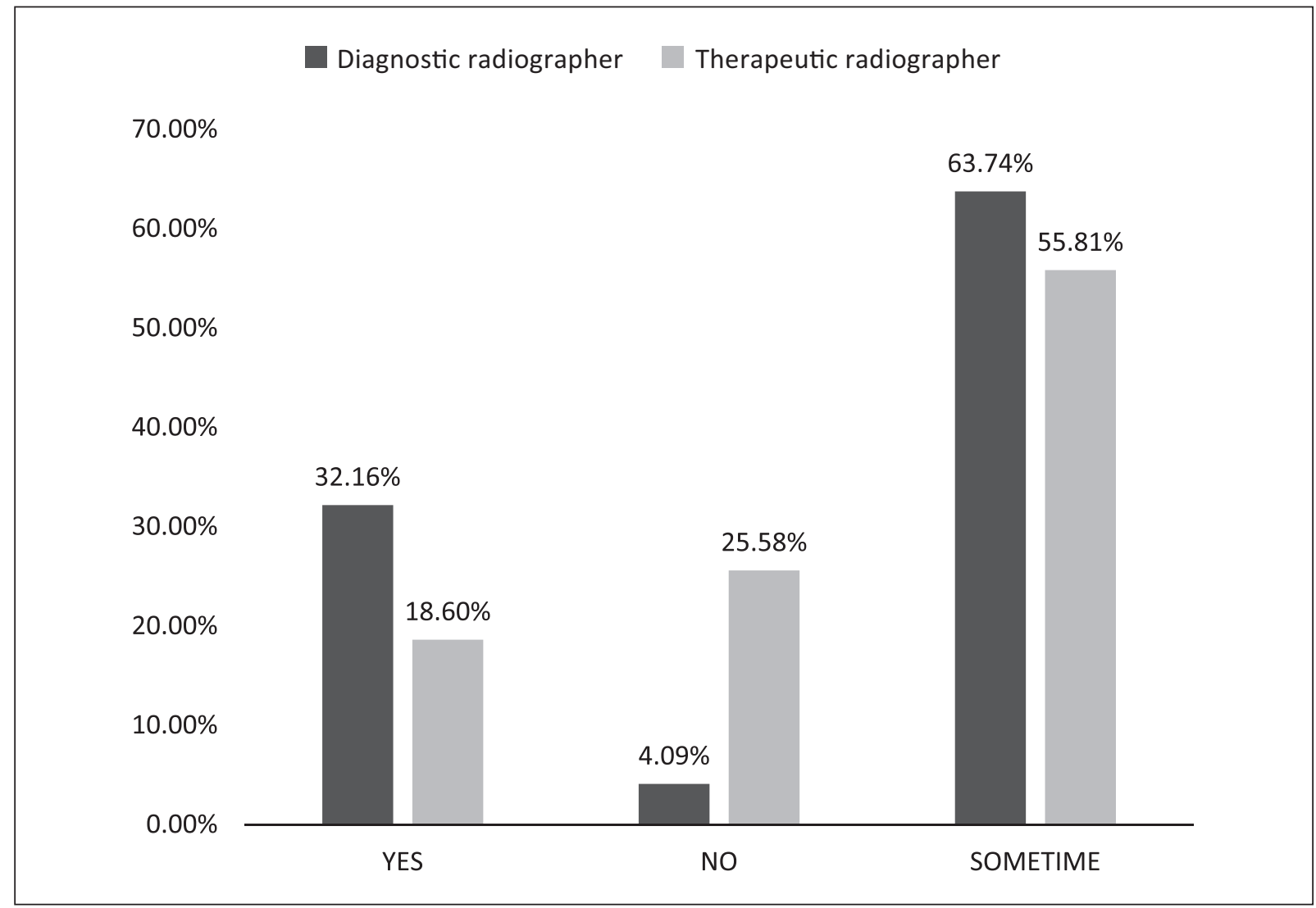

Fig. 2. Percentage of the radiographer's perception of stress during the COVID-19 pandemic in the workplace. The association exist between radiographers and the perception of stress and it was significant, $\chi^{2}(1, \mathrm{~N}=214)=21.4, p=0.000023$.

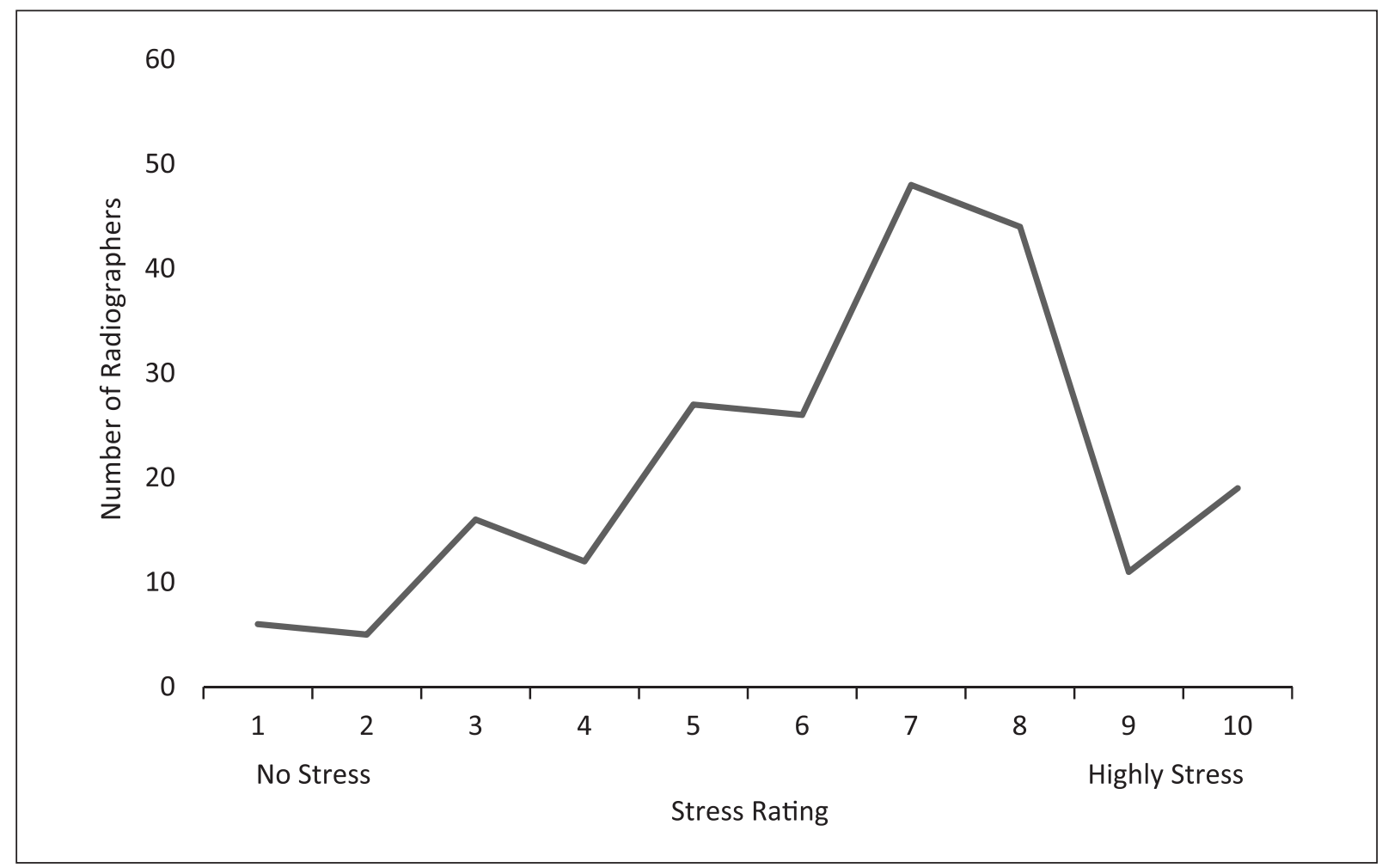

Fig. 3. The frequency of radiographers and the stress rating scale from 1 (no stress) to 10 (highly stress) during the COVID-19 pandemic. The majority of radiographers rate their level of stress between 5 to 8 . 
seem to be an excellent practice to reduce the stress among healthcare worker especially radiographers (Muller et al., 2020).

In conclusion, the impact of the COVID-19 pandemic on radiographers is mainly due to the increase in the workload, especially among diagnostic radiographers. This situation might lead to stress in the workplace. The findings of this study provide some information to aid the radiographers in terms of training, remuneration, and psychosocial support related to workplace stress during the COVID-19 pandemic.

\section{ACKNOWLEDGEMENTS}

The authors would like to express appreciation to the Malaysian Society of Radiographers (MSR) that provides database information of radiographers in Malaysia.

\section{REFERENCES}

Akudjedu, T.N., Lawal, O., Sharma, M., Elliott, J., Stewart, S., Gilleece, T., McFadden, S. \& Franklin, J.M. 2020. Impact of the COVID-19 pandemic on radiography practise: Findings from a UK radiography workforce survey. BJR Open, 2: 20200023.

Azlan, A.A., Hamzah, M.R., Sern, T.J., Ayub, S.H., Mohamad, E. 2020. Public knowledge, attitudes and practices towards COVID-19: A crosssectional study in Malaysia. PLoS ONE, 15(5): e0233668.

Das, S. \& Rajalingham, S. 2020. Personal protective equipment (PPE) and its use in COVID-19: Important facts. Indian Journal of Surgery, 82: 282-283.

Dewey, C., Hingle, S., Goelz, E. \& Linzer, M. 2020. Supporting clinicians during the COVID-19 pandemic. Annals of Internal Medicine, 172(11): 752-753.

Kooraki, S., Hosseiny, M., Myers, L. \& Gholamrezanezhad, A. 2020. Coronavirus (COVID-19) outbreak: What the department of radiology should know. Journal of the American College of Radiology, 17(4): 447e51.

Muller, A.E., Hafstad, E.V., Himmels, J.P.W., Smedslund, G., Flottorp, S., Stensland, S.O., Stroobants, S., de-Velde, S.V. \& Vist, G.E. 2020. The mental health impact of the COVID-19 pandemic on healthcare workers, and interventions to help them: A rapid systematic review. Psychiatry Research, 293: 113441.
Nguyen, L.H., Drew, D.A., Graham, M.S., Joshi, A.D., Guo, C-G, Ma, W., Mehta, R.S., Warner, E.T., Sikavi, D.R., Lo, C-H, Kwon, S., Song, M., Mucci, L.A., Stampfer, M.J., Willett, W.C., Eliassen, A.H., Hart, J.E., Chavarro, J.E., Rich-Edwards, J.W., Davies, R., Capdevila, J., Lee, K.A., Lochlainn, M.N., Varsavsky, T., Sudre, C.H., Cardoso, M.J., Wolf, J., Spector, T.D., Ourselin, S., Steves, C.J., Chan, A.T. 2020. Risk of COVID19 among frontline healthcare workers and the general community: A prospective cohort study. Lancet Public Health, 5: e475-83.

Olum, R., Chekwech, G., Wekha, G., Nassozi, D.R. \& Bongomin, F. 2020. Coronavirus Disease-2019: Knowledge, attitude, and practices of health care workers at Makerere University Teaching Hospitals, Uganda. Frontiers in Public Health, 8: 181.

Shoja, E., Aghamohammadi, V., Bazyar, H., Moghaddam, H.R., Nasiri, K., Dashti, M., Choupani, A., Garaee, M., Aliasgharzadeh, S. \& Asgari, A. 2020. COVID-19 effects on the workload of Iranian healthcare workers. BMC Public Health 20: 1636.

Shoji, K., Lesnierowska, M., Smoktunowicz, E., Bock, J., Luszczynska, A., Benight, B.C. 2015. What comes first, job burnout or secondary traumatic stress? Findings from two longitudinal studies from the U.S. and Poland. PLoS One, 10(8): $1-15$.

Tan, P.T. 2020. Saluting our COVID-19 frontliners URL https://codeblue.galencentre.org/2020/07/ 21/saluting-our-covid-19-frontliners/ (accessed 10.14.20).

Wong, L.P. \& Sam, I.C. 2011. Behavioral responses to the influenza A (H1N1) outbreak in Malaysia. Journal of Behavioral Medicine, 34(1): 23-31.

Wong, L.P. \& Sam, I.C. 2010. Temporal changes in psychobehavioral responses during the 2009 H1N1 influenza pandemic. Preventive Medicine, 51(1): 92-93.

Zervides, C., Sassi, M., Kefala-Karli, P. \& Sassis, L. 2020. Impact of COVID-19 pandemic on radiographers in the Republic of Cyprus. A questionnaire surveys. Radiography, 27(2): 419-424.

Zhou, Q. \& Zhang, X. 2020. Influence of workload, mental health and professional quality of life on healthcare workers' hand hygiene behavior in medical aid during COVID19 pandemic (Preprint).

Zhu, N., Zhang, D., Wang, W., Li, X., Yang, B., Song, J., Zhao, X., Huang, B., Shi, W., Lu, R., Niu, P., Zhan, F., Ma, X., Wang, D., Xu, W., Wu, G., Gao, G.F., Tan, W. \& China Novel Coronavirus Investigating and Research Team. 2019. A novel coronavirus from patients with pneumonia in China, New England Journal of Medicine, 382(8): $727 \mathrm{e} 33$. 